\title{
基于足端位置的六足机器人漫游地形感知与表征
}

\author{
张明路 王 哲 李满宏 张建华 陈俊杰 \\ (河北工业大学机械工程学院 天津 300130)
}

\begin{abstract}
摘要: 未知复杂地形的精准感知与量化表征长期制约着六足机器人运动性能与作业效能的本质提升。针对传统基于外部传感 的地形感知与表征方法普遍存在的感知范围局限、感知精度不足、表征效果欠佳等突出问题, 研究借鉴足式生物地形感知机 理, 充分利用足端与地形交替离散接触特性, 创新提出基于足端位置的六足机器人漫游地形感知与表征方法。通过构建时变 机体坐标系下足端位置解算模型, 解决漫游地形无序足端序列坐标高效求取难题。基于足端序列的周期化处理与矢量化描述, 建立基于周期足端位置状态的局部地形量化表征方法, 间接构建时变机体位姿与局部地形间周期映射关系。系统分析相邻周 期机体位姿间耦合约束与变换机制, 建立基于机体位姿变换的全局形貌拓扑重构方法, 以连续精准机体位姿作为参照实现周 期映射局部地形的拓扑拼接。样机实验结果表明, 基于足端位置的六足机器人地形感知与表征方法相比传统方法能够在无需增 设外部观测传感器件条件下较为精准合理的量化表征不同特征局部地形, 并实现漫游地形全局形貌的精准拓扑重构。
\end{abstract}

关键词: 六足机器人; 运动学分析; 地形感知; 量化表征; 形貌重构

中图分类号: TP242

\section{Perception and Representation of Roaming Terrain for a Hexapod Robot Based on Foot Positions}

\author{
ZHANG Minglu WANG Zhe LI Manhong ZHANG Jianhua CHEN Junjie \\ (School of Mechanical Engineering, Hebei University of Technology, Tianjin 300130)
}

\begin{abstract}
The perception and representation of unknown complex terrains restrict the raise of motion property and operational efficiency of hexapod robots for a long time. Aiming at the significant issues of traditional terrain perception and representation methods based on external sensing, such as the limitation of perception range, insufficient perception accuracy and poor representation effect, the method of perception and representation of roaming terrain for hexapod robot based on foot positions is innovatively proposed by researching on the terrain perception mechanism of multi-legged creatures and making full use of the characteristic of alternative discrete contact between feet and terrains. A computing model is constructed to efficiently obtain the coordinates of disordered foot positions in the time-varying body coordinate system. Based on the periodic processing and vectorized description of foot sequences, a local terrain representation method based on foot positions is established and the mapping relationship between the time-varying body poses and local terrain is constructed. The coupling constraint and the transformation mechanism between body poses during adjacent periods is systematically analyzed and then the global terrain reconstruction method is established. The splicing of periodic local mapping terrain by setting continuous and accurate body poses as the reference is realized. The experiment shows that this method can represent different local terrains more accurately and reasonably without additional external sensors than traditional methods and accurately reconstruct the global topography of roaming terrain.
\end{abstract}

Key words: hexapod robot; kinematics analysis; terrain perception; quantitative characterization; morphology reconstruction

0 前言

以 “步态丰富、结构冗余” 为突出特征的六足

* 国家自然科学基金(61803142, U1913211)和河北省自然科学基金 (F2021202016，F2018202210)资助项目。20201016 收到初稿, 20210303 收到修改稿
机器人运动灵活、可靠性高, 加之得益于足端与地 形 “离散接触、适时转移” 驱动本源，具备卓越的 地形适应能力，在国民经济与国防建设关键领域中 不可替代作用日益突显 ${ }^{[1-3]}$ 。地形精准感知与量化表 征作为六足机器人运动规划的基本前提, 直接影响 着未知复杂地形下机器人柔顺运动性能的本质提 
升, 根源制约着当前六足机器人的发展与应用, 始 终是六足机器人基础理论研究的重点与难点 ${ }^{[4-6]}$ 。

目前, 国内外针对六足机器人地形感知与量化 表征已开展广泛深入研究 ${ }^{[7-9]}$, 普遍借鉴 SLAM 原 理, 利用外部观测传感信息感知并重构地形三维形 貌。如 EINHORN 等 ${ }^{[10]}$ 提出采用深度相机感知局部 地形典型特征, 建立了基于定向特征视场拓扑拼接 的全局形貌重构表征方法; 赵杰等 ${ }^{[11]}$ 利用机载双目 相机采集地形视差图像, 通过特征快速匹配与点云 三角剖分初步实现了六足机器人漫游地形动态建 模; 白瑞林等 ${ }^{[12]}$ 针对双目相机点云数据匹配时间 长、表征精度低等问题, 提出了基于 ORB-SLAM2 的三维稠密地形建模方法; LOMBARD 等 ${ }^{[13]}$ 针对视 觉感知精度易受环境杂散光干扰等问题, 选用激光 雷达感知地形三维信息, 提出了基于随机三角网格 映射的局部地形量化表征方法; 刘济林等 ${ }^{[14]}$ 基于激 光点云配准融合与精简表征初步实现了地形全局形 貌感知重构; STUECKLER 等 ${ }^{[15]}$ 针对特定场景下单 一外部观测传感器件存在的感知精度局限, 提出了 融合三维激光雷达和全向 RGB-D 相机的全局地形 感知与表征方法; 李贻斌等 ${ }^{[16]}$ 通过深度融合激光雷 达和深度相机传感数据, 利用 GPR 模型动态修正点 云误差, 有效提升了基于外部传感的地形感知与表 征精度; BELTER 等 ${ }^{[17]}$ 针对外部传感器件观测针点 位姿难以精准确定导致的全局形貌表征精度欠佳问 题, 通过局部地形激光雷达和双目相机点云数据特 征匹配, 提出了基于高斯曲面逼近的全局形貌拓扑 拼接方法; FANKHAUSER 等 ${ }^{[18]}$ 创新借助四足机器 人机体位姿实时解算间接精准获取观测针点时变位 姿, 提出基于连续机体位姿参照的全局地形视觉感 知方法, 大幅提升了机器人漫游地形全局形貌重构 表征精度。

综上所述, 现有基于外部传感的地形感知与表 征方法已初步实现未知复杂地形的局部鲁棒感知与 全局形貌重构, 但仍普遍存在感知范围局限、感知 精度不足、表征效果欠佳等突出问题, 集中表现如 下: (1) 受限于机器人外部器件观测视场与自身结构 局部遮挡, 存在潜在感知盲区, 难以实现临近地形 全域感知; (2) 受限于外界环境噪声干扰与传感器件 精度特性, 感知精度易受光照、距离等因素影响, 存在非线性随机误差; (3) 受限于观测针点位姿精度 与局部地形拼接原理, 全局形貌三维重构计算繁琐, 局部地形量化表征精度欠佳。事实上, 足式生物利 用足端位置协同反馈微观感知局部地形, 借助躯体 位姿外部参照宏观评估全局形貌, 进而通过合理规
划时空离散化落足点序列, 实现未知复杂地形下动 态柔顺运动。因此, 如何借鉴生物基于肢体本征结 构位姿变换表征与时空拓扑重构的地形感知机理, 探索未知复杂地形机器人局部精准感知与全局量化 表征方法, 以满足机器人在线运动控制与再入运动 规划要求, 已成为当前六足机器人领域研究亟待解 决的关键问题。

针对上述问题, 本文区别传统基于外部传感的 地形感知与表征方法, 深度借鉴生物地形感知机理, 充分利用六足机器人运动过程中足端与地形交替离 散接触特性, 通过构建基于可测关节转角的时变机 体坐标系下足端位置解算模型, 创新提出基于无序 足端位置的局部地形精准感知方法, 旨在通过时变 并联机构精准解算建立机体位姿与局部地形间瞬时 映射关系, 解决传统方法感知局限、精度欠佳问题, 系统分析时变机体位姿间耦合约束与变换机制, 创 新建立基于机体位姿时空变换的全局形貌拓扑重构 方法, 以期基于连续精准机体位姿参照实现其映射 局部地形序列的拓扑拼接, 提升现有方法重构效率、 表征精度, 在此基础上, 从新的视角提出一套适用 于六足机器人漫游控制的地形感知与量化表征方 法, 并利用样机开展系列测试实验。

\section{1 机体坐标系下足端位置解算}

鉴于六足机器人运动过程中足端与地形始终保 持交替离散接触状态, 因而可利用各足端位置量化 表征任意时刻下机器人所处局部地形。相较于传统 基于外部传感的地形感知与表征方法, 采用足端位 置信息能够在无需增设外部观测传感器件的条件下 有效感知实际支撑地层、消除潜在感知盲区、抑制 传感误差干扰。然而, 因六足机器人支撑足端数目 与机体位置姿态呈现明显的不确定性与时变特征, 构成一时变并联机构 ${ }^{[19-20]}$, 以致用于实时表征局部 地形的足端位置求解尤为复杂关键。为此, 基于机 器人构型分析与结构约束, 采用矢量法构建出机体 坐标系下足端位置解算模型, 以利用各关节转角数 据高效求取足端坐标, 满足时变机体位姿参照下局 部地形量化表征需求。

为便于阐述时变机体坐标系下足端位置解算方 法, 以六足机器人 HEXA 为例详述。因机器人采用 典型圆周机体布置形式, 即六条结构相同的三关节 单足周向均布于机体, 故建立如图 1 所示的运动学 坐标系以利于矢量法分析求解。图中, $\Sigma C$ 为固着 于机体形心 $C$ 的机体坐标系, 其 $y$ 轴沿机体轴线指 
向前进方向, $z$ 轴垂直于机体平面背离重力方向, $x$ 轴遵循右手螺旋定则, $\Sigma W$ 为固连于地形基准的参 考坐标系, 其各轴均与机体坐标系对应轴平行同向, $\Sigma V_{i}$ 为固定于第 $i$ 足与机体连接点 $V_{i}$ 的关节坐标系, 其 $z$ 轴与机体坐标系平行同向, $y$ 轴在机体平面内 垂直于连接点与机体形心连线指向机体前进方向, $x$ 轴与机体坐标系对应轴偏角可用其绕 $z$ 轴顺时针 方向旋至两轴平行同向所需角度 $\theta_{i}$ 描述, $U_{i}$ 为第 $i$ 足足端， $\phi_{i} 、 \varphi_{i} 、 \lambda_{i}$ 分别代表第 $i$ 足躯基关节、基 股关节、股胫关节相应转角, 其零度位置与正负方 向如图所示, 机器人圆周机体半径为 $r$, 各足基节、 股节、胫节长度依次为 $L_{0} 、 L_{1} 、 L_{2}$ 。

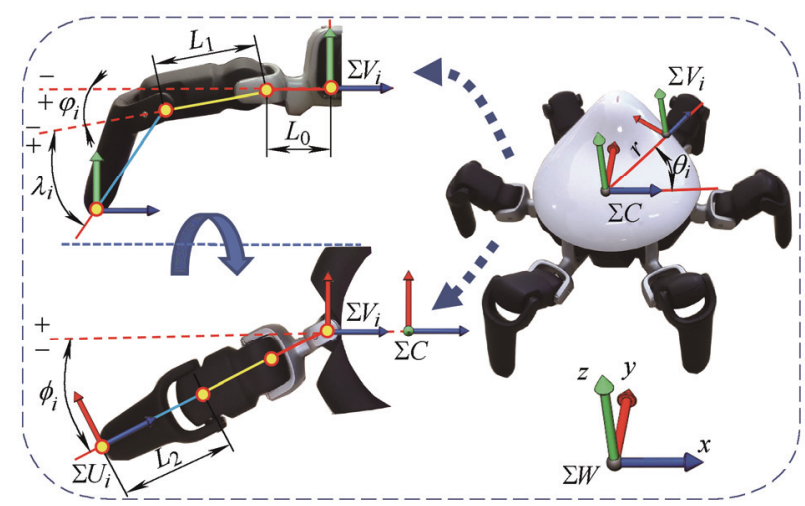

图 1 机器人运动学坐标系

定义 ${ }^{M} \boldsymbol{P}_{N}$ 为点 $N$ 在坐标系 $\Sigma M$ 中的位矢, ${ }^{M} \boldsymbol{R}_{N}$ 为坐标系 $\Sigma N$ 相对于坐标系 $\Sigma M$ 的姿态矩阵, $\left({ }^{M} x_{N},{ }^{M} y_{N},{ }^{M} z_{N}\right)$ 为点 $N$ 在坐标系 $\Sigma M$ 中的坐标。

根据矢量在坐标系间变换关系, 可知足端在机 体与参考坐标系间的位矢映射如下

$$
{ }^{W} \boldsymbol{P}_{U_{i}}={ }^{W} \boldsymbol{P}_{C}+{ }^{W} \boldsymbol{R}_{C}{ }^{C} \boldsymbol{P}_{U_{i}}
$$

因 ${ }^{W} \boldsymbol{R}_{C}$ 为单位正交阵, 其逆即为本身, 故由(1) 式可知足端在机体坐标系中的位矢如下

$$
{ }^{C} \boldsymbol{P}_{U_{i}}={ }^{W} \boldsymbol{R}_{C}\left({ }^{W} \boldsymbol{P}_{U_{i}}-{ }^{W} \boldsymbol{P}_{C}\right)
$$

鉴于机器人运动学坐标系中同时存在如下位矢 变换关系

$$
\left\{\begin{array}{c}
{ }^{W} \boldsymbol{P}_{U_{i}}={ }^{W} \boldsymbol{P}_{V_{i}}+{ }^{W} \boldsymbol{R}_{V_{i}}{ }^{V_{i}} \boldsymbol{P}_{U_{i}} \\
{ }^{W} \boldsymbol{P}_{V_{i}}={ }^{W} \boldsymbol{P}_{C}+{ }^{W} \boldsymbol{R}_{C}{ }^{C} \boldsymbol{P}_{V_{i}}
\end{array}\right.
$$

将式(3)代入式(2), 化简可知足端在机体坐标系 中的位矢亦可表示如下

$$
{ }^{C} \boldsymbol{P}_{U_{i}}={ }^{C} \boldsymbol{P}_{V_{i}}+{ }^{C} \boldsymbol{R}_{V_{i}}{ }^{V_{i}} \boldsymbol{P}_{U_{i}}
$$

由式(4)可知, 足端在机体坐标系中的位矢 ${ }^{C} \boldsymbol{P}_{U_{i}}$ 由足端在关节坐标系中的位矢 ${ }^{V_{i}} \boldsymbol{P}_{U_{i}}$ 、各足与机体连
接点在机体坐标系中的位矢 ${ }^{C} \boldsymbol{P}_{V_{i}}$ 和关节坐标系相对 机体坐标系的姿态矩阵 ${ }^{C} \boldsymbol{R}_{V_{i}}$ 共同决定。由于 ${ }^{C} \boldsymbol{P}_{V_{i}}$ 和 ${ }^{C} \boldsymbol{R}_{V_{i}}$ 取决于机体固有结构参数, 具体如式(5)、(6) 所示, 式中 $f$ 为因机体左右两侧足端位于机体坐标 系不同象限所附加的方向系数, 左侧为 -1 , 右侧则 取 1 , 因而足端在机体坐标系下的位矢实质上决定 于足端在关节坐标系中的位矢, 即关节坐标系下足 端坐标

$$
\begin{aligned}
{ }^{C} \boldsymbol{P}_{V_{i}} & =\left[\begin{array}{lll}
f r \cos \theta_{i} & f r \sin \theta_{i} & 0
\end{array}\right]^{\mathrm{T}} \\
{ }^{C} \boldsymbol{R}_{V_{i}} & =\left[\begin{array}{ccc}
\cos \theta_{i} & -\sin \theta_{i} & 0 \\
\sin \theta_{i} & \cos \theta_{i} & 0 \\
0 & 0 & 1
\end{array}\right]
\end{aligned}
$$

鉴于机器人六足结构相同, 且存在如图 2 所示 的简明几何关系, 故可利用几何投影变换便捷列写 出各足关节转角与其关节坐标系下足端坐标间映射 关系如式(7)所示。

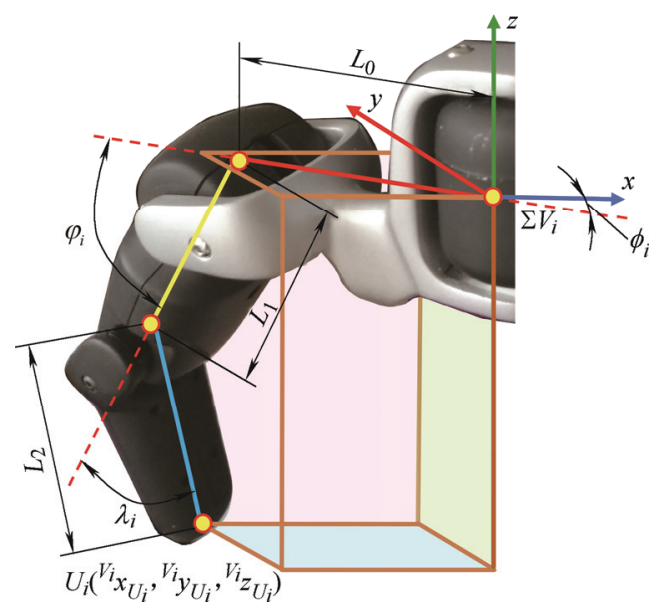

图 2 机器人单足几何关系

$$
\left\{\begin{array}{l}
f\left[L_{0}+L_{1} \cos \varphi_{i}+L_{2} \cos \left(\varphi_{i}+\lambda_{i}\right)\right] \cos \phi_{i}={ }^{V_{i}} x_{U_{i}} \\
{\left[L_{0}+L_{1} \cos \varphi_{i}+L_{2} \cos \left(\varphi_{i}+\lambda_{i}\right)\right] \sin \phi_{i}={ }^{V_{i}} y_{U_{i}}} \\
-L_{1} \sin \varphi_{i}-L_{2} \sin \left(\varphi_{i}+\lambda_{i}\right)={ }^{V_{i}} z_{U_{i}}
\end{array}\right.
$$

结合式(4) (7), 利用机器人各足关节转角及其 固有结构参数可快速解算出时变机体坐标系下足端 坐标如下

$$
\left[\begin{array}{c}
{ }^{C} x_{U_{i}} \\
{ }^{C} y_{U_{i}} \\
{ }^{C} z_{U_{i}}
\end{array}\right]=\left[\begin{array}{c}
f r \cos \theta_{i}+{ }^{V_{i}} x_{U_{i}} \cos \theta_{i}-{ }^{V_{i}} y_{U_{i}} \sin \theta_{i} \\
f r \sin \theta_{i}+{ }^{V_{i}} x_{U_{i}} \sin \theta_{i}+{ }^{V_{i}} y_{U_{i}} \cos \theta_{i} \\
{ }^{V_{i}} z_{U_{i}}
\end{array}\right]
$$

至此, 基于矢量法, 结合单足结构约束分析, 
建立了机体坐标系下足端位置解算模型, 通过利用 各关节实时转角高效求取支撑足端瞬时坐标, 能够 有效满足时变机体位姿参照下局部地形即时量化表 征要求。事实上, 由于足端位置能够真实反应松软 地质下实际支撑地形, 关节转角无需增设外部传感 器件即可精确获取, 通过时变并联机构解算校正可 有效抑制感知盲区和传感误差。因此, 以机体平面 作为参照基准, 利用实际支撑足端位置信息, 量化 表征机器人漫游局部地形合理性、实用性、精准性 更具优势。

\section{2 基于足端位置的局部地形量化表征}

六足机器人通常以自由步态漫游于未知复杂地 形, 其支撑足端数目和位置呈现明显的随机与时变 特征, 如何充分利用杂乱无序的足端位置在机体坐 标系下精准量化表征局部地形面临巨大挑战, 其间 需重点解决三大难题: (1) 无序足端位置的统一描 述; (2) 关键地形特征点的合理选取; (3) 局部地形 的精准量化表征。为此, 创新采用足端位置矢量系 统描述时变足端位置与支撑状态, 充分综合各足端 位置信息制定关键地形特征点迭代篎选规则, 以利 用特征点构造的微小特征平面精准量化表征机器人 漫游局部地形。

(1) 无序足端位置的统一描述。

机器人漫游于未知复杂地形时其足端位置及 支撑状态虽表面呈现明显的无序性与随机性, 但 本质上仍严苛遵循固有的时序和空间约束关系。 如何基于足端位置状态时空转换机制, 建立无序 足端位置状态序列描述方法是实现局部地形量化 表征的重要基础。鉴于机器人所处局部地形的感 知表征仅与支撑足端位置直接相关, 且漫游过程 中支撑足端位置状态时域内呈现非定期间歇变 化, 故可以存在支撑足端位置状态发生变化时刻 为分界点, 将足端位置状态序列离散为若干单元 状态周期, 则各周期内支撑足端位置状态均保持 固定不变。受此启发, 通过足端位置状态序列的 周期化处理, 仅利用各支撑足端坐标即可精简描 述序列中任意周期足端位置状态, 故制定出如下 足端位置状态描述规则:

描述规则 1: 定义机器人右前足为 1 号足, 按 顺时针方向将六足依次顺序编号, 考虑后续地形特 征点迭代篮选处理需要, 对 $1 、 2 、 3 、 4$ 号足重复编
号, 具体如图 3 所示。

○摆动足端 0 支撑足端

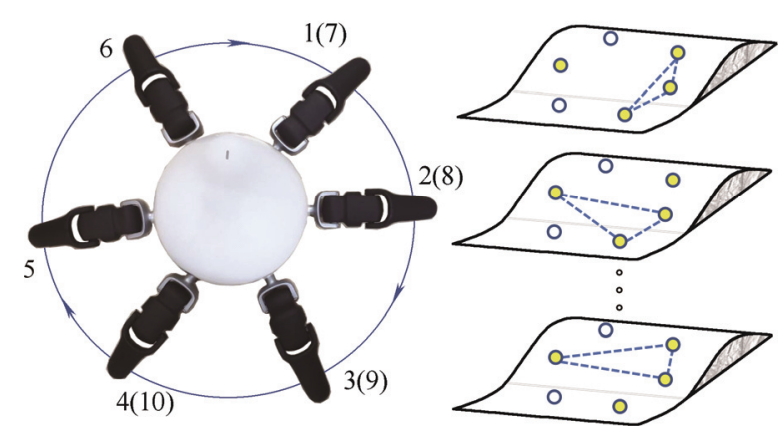

图 3 机器人足端编号

描述规则 2: 定义 $S_{\mathrm{sp}}$ 和 $S_{\mathrm{sw}}$ 分别为各周期支撑 足端和摆动足端编号构成的集合，则第 $i$ 足足端位 置状态 $p_{i}$ 可依据式(9)描述, 当足端处于支撑相时其 值赋为机体坐标系下足端坐标, 而当处于摆动相时 则暂记为 $(0,0,0)$ 。

$$
p_{i}=\left\{\begin{array}{cc}
\left({ }^{C} x_{U_{i}},{ }^{C} \mathrm{y}_{U_{i}},{ }^{C} z_{U_{i}}\right) & i \in S_{\mathrm{sp}} \\
(0,0,0) & i \in S_{\mathrm{sw}}
\end{array}\right.
$$

基于上述规则, 任意周期内机器人足端位置状 态均可用足端位置矢量 $\boldsymbol{P}\left(\begin{array}{llllll}p_{1} & p_{2} & p_{3} & p_{4} & p_{5} & p_{6}\end{array}\right)$ 统 一描述。由于足端位置矢量可直接精简描述各周期 支撑足端坐标及摆动足端编号，同时通过相邻周期 的矢量参数对比亦能间接获悉足端位置状态转换情 况，因而极适用于后续地形量化表征与拓扑重构的 分析处理。此外, 考虑同一周期内机器人足端位置 状态与所处局部地形间存在瞬时映射关系, 描述方 法通过引入时域内周期化处理机制, 在将无序足端 序列离散为若干单元足端位置状态周期以简化表征 的同时, 亦可同步实现机器人漫游全局地形向周期 映射局部地形的自然分割。

(2) 关键地形特征点的合理选取。

机器人足端位置状态与所处局部地形间瞬时映 射关系理论上仅能依据相应周期内支撑足端位置解 算构建。然而, 受限于离散足端支撑数目、位置分 布等因素制约，少量稀疏分布的支撑足端所蕴含的 局部地形特征信息较为有限。因此，如何充分利用 相对局限的支撑足端位置信息, 通过制定恰当合理 的迭代篎选规则, 遴选出科学有效的地形特征点是 实现局部地形精准量化表征的重点与难点。

机器人通常以多足交替离散接触形式漫游于未 知复杂地形, 但任意时刻内均将保持三足及以上支 撑以确保动静态运动稳定 ${ }^{[21-22]}$ 。当机器人处于三足 支撑时，由于支撑足端所采集到的地形信息极为有 
限, 故可无需经篮选处理, 直接将三个支撑足端视 为关键地形特征点。如若存在至少四足支撑, 则可 依据如下迭代篎选原则, 通过充分综合各支撑足端 位置信息, 合理选取局部地形关键特征点：依次以 各支撑足端为起点, 按足端编号增序方向, 顺序选 取三个相邻支撑足端为同一足端分组, 则各分组足 端均将构成一三角形, 暂记各足端三角形重心为关 键地形特征点, 具体如图 4 所示。由于各地形特征 点通过遍历组合和隐含加权方式, 均已充分综合任 意三个相邻支撑足端坐标信息, 利用 $z$ 轴坐标分量 初步均衡地形高程误差, 借助 $x$ 轴和 $y$ 轴分量自适 应调整特征点位置分布, 因而能够较为合理准确的 综合表征微小局部地形整体特征。

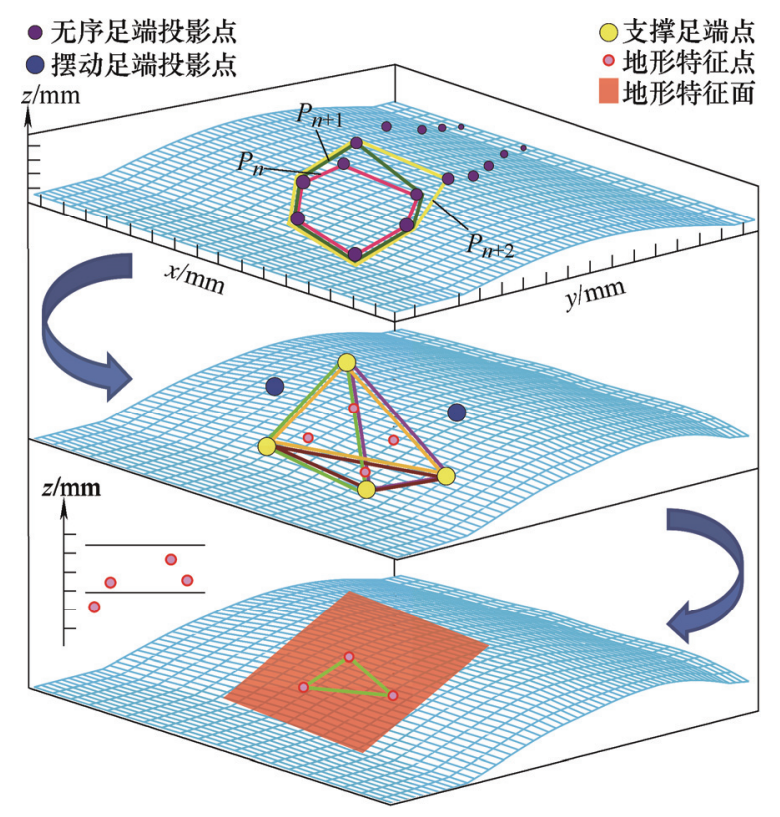

图 4 局部地形量化表征原理

基于上述原则, 如何依据各周期时变足端位置 状态快速有效笁选出符合要求的足端分组已成为地 形特征点合理选取的关键。为便于利用足端位置矢 量程式化求取各周期足端分组, 可建立如式(10)所 示的足端分组集合, 式中 $h 、 j 、 k$ 均为足端编号且满 足 $h<j<k$ 。由于足端分组集合 $Q_{S}$ 中已包含所有可 能存在的足端分组, 故可通过制定恰当合理的迭代 篮选规则, 剔除各类不满足约束的足端分组, 以最 终篎选出符合要求的足端分组

$$
Q_{s}=\{(h, j, k) \mid h \in[1,6], j \in[2,9], k \in[3,10]\}
$$

综合考虑足端支撑状态、位置分布等因素, 制 定出如下足端分组篎选规则:

篮选规则 1: 因地形特征点需基于支撑足端坐 标解算, 故任意足端分组中三个足端均应处于支撑 相，即 $h 、 j 、 k$ 均须属于支撑足端编号集合 $S_{\mathrm{sp}}$ 。
笁选规则 2: 因任意足端分组均须为三个相邻 支撑足端，故在足端编号 $h$ 和 $k$ 间应恰好存在 $k-h-2$ 个摆动足端, 且其足端编号不应为 $j$ 。

篮选规则 3: 针对分组内三足均位于机体同 侧特殊情况, 因其足端三角形构成面域相对较小 以致蕴含地形信息较为局限, 故当机器人处于四 足以上支撑时, 可剔除此类分组, 而当恰好位于 四足支撑时, 为确保至少获取三组有效足端分组 以满足地形特征点求取数量要求, 则建议保留 处理。

综合以上篎选规则, 所有符合地形特征点迭代 笁选原则的足端分组均需要满足式(11)所示约束。

$$
\left\{\begin{array}{l}
\{h, j, k\} \subset S_{\mathrm{sp}} \\
\{h+n \mid n \in[1, k-h-1], h+n \neq j\} \subset S_{\mathrm{sw}} \\
\left\{(1,2,3),(4,5,6)|| S_{\mathrm{sp}} \mid>4\right\} \not \subset Q_{S}
\end{array}\right.
$$

经分析可知, 除机器人处于三足支撑时直接选 取三个支撑足端作为关键地形特征点外, 其余支撑 状态下均可依据式(10)和式(11)程式化篎选出四个 符合要求的足端分组 $\widehat{Q}_{s}\left(h_{s}, j_{s}, k_{s}\right)(s=1,2,3,4)$, 则 各分组对应足端三角形重心 $G_{s}\left(x_{G_{s}}, y_{G_{s}}, z_{G_{s}}\right)$ 即为拟 求取的关键地形特征点。鉴于各支撑足端坐标已在 足端位置矢量中明确列写, 故可依据几何重心解算通 式便捷求得机体坐标系下地形特征点坐标如下

$$
\left[\begin{array}{c}
x_{G_{s}} \\
y_{G_{s}} \\
z_{G_{s}}
\end{array}\right]=\left[\begin{array}{l}
\left({ }^{C} x_{U_{h_{s}}}+{ }^{C} x_{U_{j_{s}}}+{ }^{C} x_{U_{k_{s}}}\right) / 3 \\
\left({ }^{C} y_{U_{h_{s}}}+{ }^{C} y_{U_{J_{s}}}+{ }^{C} y_{U_{k_{s}}}\right) / 3 \\
\left({ }^{C} z_{U_{h_{s}}}+{ }^{C} z_{U_{J_{s}}}+{ }^{C} z_{U_{k_{s}}}\right) / 3
\end{array}\right]
$$

至此, 根据足端位置矢量可快速解算出各关键 地形特征点坐标以用于局部地形量化表征。由于地 形特征点选取方法可根据足端位置状态分布通过动 态遍历形式合理构建有效足端分组，同时能够借助 重心隐含加权方式充分综合任意三个相邻支撑足端 坐标，不仅可利用 $z$ 轴坐标分量初步均衡地形高程 误差，以有效抑制地形局部凹坑或凸起对整体高程 表征影响, 而且能综合 $x$ 轴和 $y$ 轴分量自适应调整 特征点位置分布，以向支撑足端构成面域中心拓展 延伸, 因而能够较为合理准确的综合表征微小局部 地形整体特征。

（3）局部地形的精准量化表征。

机器人以自由步态漫游于未知复杂地形时将产 生大量无序足端位置状态序列, 基于时域内序列的 周期化处理，各周期足端位置状态所映射的局部地 
形区域事实上极为狭小。因而, 通过充分综合各支 撑足端位置信息, 利用迭代篮选出的关键地形特征 点合理构造局部地形特征平面, 能够在较高精度要 求下量化表征微小局部地形。

如前所述, 当机器人处于三足支撑时, 支撑足 端即可视为关键地形特征点, 故可直接利用三个支 撑足端坐标解析构建局部地形特征平面。而当机器 人处于其他支撑状态时, 依据地形特征点迭代篮选 规则, 则均将获取四个关键地形特征点。因此, 如 何从中再次遴选出三个更为合理的地形特征点以用 于局部地形特征平面构建是需解决的又一难题。鉴 于微小区域范围内局部地形特征主要体现于高程变 化, 故可基于地形特征点 $z$ 轴坐标分量遴选三个高 程分布相对集中的地形特征点, 具体可参照式(13) 程式化选取, 式中 $z_{\text {max }} 、 z_{2 \mathrm{nd}} 、 z_{3 \mathrm{rd}} 、 z_{\text {min }}$ 分别为按 数值由大到小顺序排列的四个地形特征点 $z$ 轴坐标 分量

$$
\left|z_{\text {max }}-z_{3 \mathrm{rd}}\right|>\left|z_{\text {min }}-z_{2 \text { nd }}\right|
$$

如若式(13)成立, 则表明 $z_{\text {max }}$ 对应的地形特征 点高程偏离较大, 依据遴选规则建议剔除, 否则需 舍弃 $z_{\min }$ 对应的地形特征点。此时, 可将最终遴选 出的三个关键地形特征点(包括机器人三足支撑时 的三个支撑足端) 分别标记为 $\widehat{G}_{1} 、 \widehat{G}_{2} 、 \widehat{G}_{3}$, 则根据 平面解析方程可便捷求得机体坐标系下局部地形特 征平面方程及法矢量如下

$$
\left[\begin{array}{lll}
x & y & z
\end{array}\right] \boldsymbol{n}-D=0
$$

$$
\begin{aligned}
& \boldsymbol{n}=\left[\begin{array}{l}
\left(y_{\widehat{G}_{2}}-y_{\widehat{G}_{1}}\right)\left(z_{\widehat{G}_{3}}-z_{\widehat{G}_{1}}\right)-\left(z_{\widehat{G}_{2}}-z_{\widehat{G}_{1}}\right)\left(y_{\widehat{G}_{3}}-y_{\widehat{G}_{1}}\right) \\
\left(x_{\widehat{G}_{3}}-x_{\widehat{G}_{1}}\right)\left(z_{\widehat{G}_{2}}-z_{\widehat{G}_{1}}\right)-\left(x_{\widehat{G}_{2}}-x_{\widehat{G}_{1}}\right)\left(z_{\widehat{G}_{3}}-z_{\widehat{G}_{1}}\right) \\
\left(x_{\widehat{G}_{2}}-x_{\widehat{G}_{1}}\right)\left(y_{\widehat{G}_{3}}-y_{\widehat{G}_{1}}\right)-\left(x_{\widehat{G}_{3}}-x_{\widehat{G}_{1}}\right)\left(y_{\widehat{G}_{2}}-y_{\widehat{G}_{1}}\right)
\end{array}\right] \\
& D=\left[\begin{array}{lll}
x_{\widehat{G}_{1}} & y_{\widehat{G}_{1}} & z_{\widehat{G}_{1}}
\end{array}\right] \boldsymbol{n}
\end{aligned}
$$

至此, 基于无序足端位置状态序列的周期化 处理与矢量化描述, 通过制定合理可行的地形特 征点迭代篎选规则以充分综合各足端位置信息, 利用程式化遴选出的关键地形特征点成功构建 出了可量化表征微小局部地形的地形特征平面。 由于机器人各周期位置状态所映射的局部地形 区域极为狭小, 同时各地形特征点通过遍历组合 和隐含加权方式已合理均衡高程误差和位置分 布, 因而基于足端位置状态程式化构建的局部地 形特征平面可在较高精度要求下合理量化表征 任意周期映射局部地形。

\section{3 基于机体位姿的全局形貌拓扑重构}

机器人漫游过程中产生的大量无序足端序列理 论上可有效表征其行经地形全局形貌，通过引入时 域内序列周期化处理机制, 在解决无序足端序列离 散化周期描述的同时亦同步实现了全局地形向周期 映射局部地形的自然分割。前文基于足端位置状态 的矢量化描述，通过制定合理有效的地形特征点迭 代篎选规则, 利用各周期足端位置状态已在机体坐 标系下程式化构建出可精准量化表征局部地形的地 形特征平面, 间接建立了各周期机体位姿与局部地 形间瞬时映射关系。然而, 机器人漫游于未知复杂 地形时机体位姿将呈现明显的时变特征, 以致固着 于机体形心的机体坐标系亦会随之动态调整。因此, 如何将时变机体坐标系下构造的各周期地形特征平 面统一转换至同一参考坐标系并进行合理裁剪拼接 已成为全局形貌拓扑重构的关键。事实上, 机器人 任意两相邻周期足端位置状态中均存在共用支撑足 端以确保动静态运动稳定, 故可以共用支撑足端作 为参照基准, 利用足端与机体间固有的约束关系以 及足端在不同机体坐标系中的位矢变换, 间接获取 相邻两周期机体位姿及其坐标系变换关系。受此启 发, 通过系统分析相邻周期机体位姿间耦合约束与 变换机制, 创新建立基于机体位姿时空变换的全局 形貌拓扑重构方法, 以期基于连续精准机体位姿参 照实现其周期映射局部地形的拓扑拼接, 解决漫游 地形全局形貌表征重构难题。

定义第 $n$ 个周期内机器人足端位置状态为 $\boldsymbol{P}_{n}$, 并记 $\boldsymbol{P}_{0}$ 为初始足端位置状态, 则依据时域内足端序 列周期化处理机制, 当存在任意足端发生相位变化 时, 足端位置状态即将转入新的周期 $\boldsymbol{P}_{n+1}$ 。为便于 以 $\boldsymbol{P}_{n}$ 和 $\boldsymbol{P}_{n+1}$ 为例简述相邻周期机体位姿及其坐标系 间变换关系, 建立了如图 5 所示的时变运动学坐标 系。图中, $\Sigma C_{n}$ 和 $\Sigma C_{n+1}$ 分别为 $\boldsymbol{P}_{n}$ 和 $\boldsymbol{P}_{n+1}$ 对应周期内 固着于机体形心的机体坐标系, $U_{s}$ 为两相邻周期内 任一共用支撑足端, 而 $\Sigma U_{s}^{n}$ 和 $\Sigma U_{s}^{n+1}$ 则为以 $U_{s}$ 为原 点构建的相应周期内足端坐标系, 其各轴均分别与 $\Sigma C_{n}$ 和 $\Sigma C_{n+1}$ 对应轴平行同向。

基于矢量在坐标系间的变换关系，可知机体形 心 $C_{n+1}$ 在足端坐标系 $\Sigma U_{s}^{n}$ 和机体坐标系 $\Sigma C_{n}$ 间的 位矢映射如下

$$
{ }^{C_{n}} \boldsymbol{P}_{C_{n+1}}={ }^{C_{n}} \boldsymbol{P}_{U_{s}}+{ }^{C_{n}} \boldsymbol{R}_{U_{s}^{n}}{ }^{U_{s}^{n}} \boldsymbol{P}_{C_{n+1}}
$$

同理, 运动学坐标系中亦存在如下位矢变换 


$$
{ }^{U_{s}^{n}} \boldsymbol{P}_{C_{n+1}}={ }^{U_{s}^{n}} \boldsymbol{P}_{U_{s}}+{ }^{U_{s}^{n}} \boldsymbol{R}_{U_{s}^{n+1}} U_{s}^{n+1} \boldsymbol{P}_{C_{n+1}}
$$

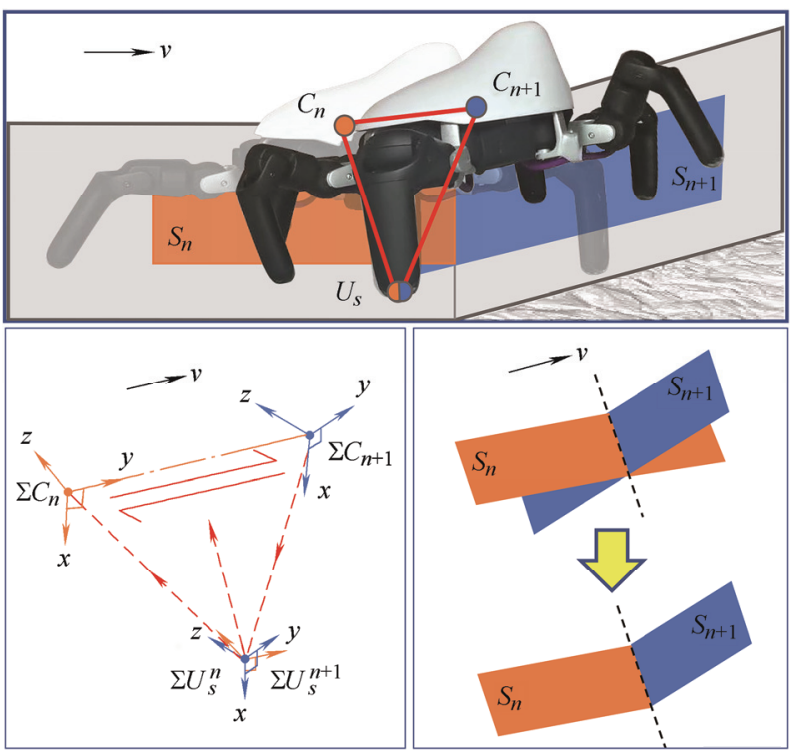

图 5 全局形貌拓扑重构原理

由于 $\Sigma U_{s}^{n}$ 与 $\Sigma C_{n}$ 各轴均平行同向, 因而 ${ }^{C_{n}} \boldsymbol{R}_{U_{s}^{n}}$ 恒为单位阵, 同时考虑 $U_{s}$ 本身即为 $\Sigma U_{s}^{n}$ 原点, 故 ${ }^{U_{s}^{n}} \boldsymbol{P}_{U_{s}}$ 实为零矢量。由此, 结合式(15)、(16)可知相 邻周期内机体位置间变换关系可表示如下

$$
{ }^{C} \boldsymbol{P}_{C_{n+1}}={ }^{C_{n}} \boldsymbol{P}_{U_{s}}+{ }^{U_{s}^{n}} \boldsymbol{R}_{U_{s}^{n+1}} U_{s}^{n+1} \boldsymbol{P}_{C_{n+1}}
$$

分析式(17)可知, ${ }^{C_{n}} \boldsymbol{P}_{U_{s}}$ 和 ${ }^{C_{n+1}} \boldsymbol{P}_{U_{s}}$ 可由式(8)利用 机器人关节转角实时解算, 同时考虑 $U_{s}$ 和 $C_{n+1}$ 两点 间位矢的相对性, ${ }^{U_{s}^{n+1}} \boldsymbol{P}_{C_{n+1}}$ 可通过取反 ${ }^{C_{n+1}} \boldsymbol{P}_{U_{s}}$ 快速获 取。由于各周期内机体坐标系与足端坐标系各轴均 平行同向, 因而相邻周期内两坐标系的姿态变化将 始终保持一致, 即存在 ${ }^{U_{s}^{n}} \boldsymbol{R}_{U_{s}^{n+1}}$ 恒等于 ${ }^{C_{n}} \boldsymbol{R}_{C_{n+1}}$ 。事实 上, 相邻周期内机体及其坐标系的姿态变化可借鉴 并联机构相关理论利用共用支撑足端与时变姿态机 体间固有的约束关系解算获取, 但考虑到全局形貌 在线重构对计算效率的严苛要求, 可利用机器人固 有陀螺仪的传感数据直接解算。定义 $\Sigma C_{n+1}$ 相对于 $\Sigma C_{n}$ 的标准欧拉角分别为 $\Delta \alpha_{n} 、 \Delta \beta_{n} 、 \Delta \gamma_{n}$, 则相 邻周期内机体姿态间变换矩阵可表示如下

$$
{ }^{C_{n}} \boldsymbol{R}_{C_{n+1}}=\left[\begin{array}{ccc}
r_{11} & r_{12} & r_{13} \\
r_{21} & r_{22} & r_{23} \\
r_{31} & r_{32} & r_{33}
\end{array}\right]
$$

式中, $r_{11}=\mathrm{c} \Delta \alpha_{n} \mathrm{c} \Delta \beta_{n}, r_{12}=\mathrm{c} \Delta \alpha_{n} \mathrm{~s} \Delta \beta_{n} \mathrm{~s} \Delta \gamma_{n}-\mathrm{s} \Delta \alpha_{n} \mathrm{c} \Delta \gamma_{n}$ $, r_{13}=\mathrm{c} \Delta \alpha_{n} \mathrm{~s} \Delta \beta_{n} \mathrm{c} \Delta \gamma_{n}+\mathrm{s} \Delta \alpha_{n} \mathrm{~s} \Delta \gamma_{n}, r_{21}=\mathrm{s} \Delta \alpha_{n} \mathrm{c} \Delta \beta_{n}, r_{22}=$ $\mathrm{s} \Delta \alpha_{n} \mathrm{~s} \Delta \beta_{n} \mathrm{~s} \Delta \gamma_{n}+\mathrm{c} \Delta \alpha_{n} \mathrm{c} \Delta \gamma_{n}, r_{23}=\mathrm{s} \Delta \alpha_{n} \mathrm{~s} \Delta \beta_{n} \mathrm{c} \Delta \gamma_{n}-\mathrm{c} \Delta \alpha_{n}$ $\mathrm{s} \Delta \gamma_{n}, r_{31}=-\mathrm{s} \Delta \beta_{n}, r_{32}=\mathrm{c} \Delta \beta_{n} \mathrm{~s} \Delta \gamma_{n}, r_{33}=\mathrm{c} \Delta \beta_{n} \mathrm{c} \Delta \gamma_{n}$

根据式(17)、(18)可知，相邻周期内机体位置间 变换关系如下

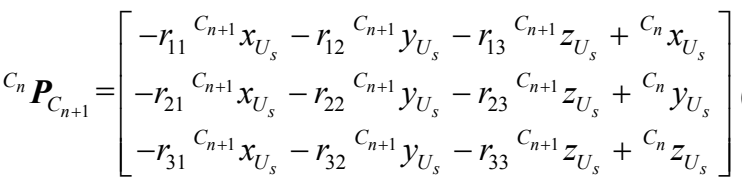

综合式(18)、(19)可知，任意两相邻周期内机体 位姿及其坐标系间的位姿变化矩阵如下

$$
{ }^{C_{n}} \boldsymbol{T}_{C_{n+1}}=\left[\begin{array}{cc}
{ }^{C_{n}} \boldsymbol{R}_{C_{n+1}} & { }^{C_{n}} \boldsymbol{P}_{C_{n+1}} \\
0 & 1
\end{array}\right]
$$

根据式(20)所示递推关系，可知任意周期内机 体坐标系 $\Sigma C_{n}$ 相对于初始参考坐标系 $\Sigma C_{0}$ 的位姿变 换矩阵如下

$$
{ }^{C_{0}} \boldsymbol{T}_{C_{n}}={ }^{C_{0}} \boldsymbol{T}_{C_{1}}{ }^{C_{1}} \boldsymbol{T}_{C_{2}} \ldots{ }^{C_{n-2}} \boldsymbol{T}_{C_{n-1}}{ }^{C_{n-1}} \boldsymbol{T}_{C_{n}}
$$

依据式(21)可便捷提取出 ${ }^{C_{0}} \boldsymbol{R}_{C_{n}}$ 和 ${ }^{C_{0}} \boldsymbol{P}_{C_{n}}$, 如若 将第 $n$ 个周期内根据足端位置状态 $\boldsymbol{P}_{n}$ 程式化遴选出 的三个地形特征点标记为 $\widehat{G}_{s}^{n}(s=1,2,3)$, 可求得其 在初始参考坐标系 $\Sigma C_{0}$ 中的坐标如下

$$
\left[\begin{array}{c}
{ }^{C_{0}} x_{\widehat{G}_{s}^{n}} \\
{ }^{C_{0}} y_{\widehat{G}_{s}^{n}} \\
{ }^{C_{0}} z_{\widehat{G}_{s}^{n}}
\end{array}\right]={ }^{C_{0}} \boldsymbol{R}_{C_{n}}\left[\begin{array}{c}
x_{\widehat{G}_{s}^{n}} \\
y_{\widehat{G}_{s}^{n}} \\
z_{\widehat{G}_{s}^{n}}
\end{array}\right]+{ }^{C_{0}} \boldsymbol{P}_{C_{n}}
$$

利用式(22)可将在时变机体坐标系下解算获取 的各周期地形特征点坐标统一转换至初始参考坐标 系，从而能够参照式(14)在同一坐标系下构造出各 周期映射局部地形特征平面。然而, 由于地形特征 平面数量极多且其边界难以有效确定, 以致如何有 效裁剪拼接各微小特征平面已成为实现全局形貌表 征重构的关键。鉴于任意两相邻周期足端位置状态 中均将存在共用支撑足端，因而基于支撑足端位置 信息构造的两相邻周期地形特征平面必然相交，故 可以其交线为界裁剪拼接各周期地形特征平面。基 于此, 综合考虑拓扑关系、计算效率、表征精度等 因素，制定出各周期地形特征平面拓扑拼接规则如 下：在初始参考坐标系下，定义 $S_{n}$ 为利用前 $n$ 个周 期地形特征平面裁剪拼接出的复合表面, $S_{n+1}$ 为基 于第 $n+1$ 个周期足端位置状态 $\boldsymbol{P}_{n+1}$ 构造出的地形特 征平面, 如若 $S_{n}$ 和 $S_{n+1}$ 两面交线封闭, 则舍弃 $S_{n}$ 交 线内部区域，摒弃 $S_{n+1}$ 交线外部区域; 反之，不封 闭交线将会参照机体运动方向将各面分割成两个区 域, 此时则可舍弃 $S_{n}$ 运动同向区域, 摒弃 $S_{n+1}$ 运动 反向区域，如图 5 所示。此外，考虑各周期地形特 征平面在机体宽度方向的表征精度呈现由中心向两 
侧逐渐降低趋势, 故可参照关键地形特征点分布通 过设定动态阈值方式限定各周期特征平面宽度。基 于上述原则, 按照时序依次裁剪各周期地形特征平 面, 利用保留的特征平面区域即可拓扑重构出机器 人漫游地形全局形貌。

至此, 基于相邻周期机体位姿间耦合约束与变换 机制深度剖析, 利用共用支撑足端坐标和机体姿态传 感数据构建了相邻周期机体位姿递推关系, 通过将时 变机体坐标系下构造的各周期地形特征平面统一转 换至同一参考坐标系并制定合理可行的特征平面拓 扑拼接规则, 实现了机器人漫游地形全局形貌的拓扑 重构。由于基于机体位姿的全局形貌拓扑重构方法主 要基于时变并联机构解算获取连续精准的机体位姿 作为局部地形拓扑拼接的参照基准, 依据拓扑拼接规 则裁剪获取的微小特征平面亦能更为精准的表征各 周期映射局部地形，同时伴随漫游进程的持续开展， 利用丰富的新增足端位置状态序列可持续提升全局 形貌拓扑重构精度, 因而相较于传统基于外部传感的 地形重构方法理论上具备更为优异的表征精度。

\section{4 局部地形表征与全局形貌重构实验}

机器人实验平台如图 6 所示, 通过依次开展局 部地形量化表征和全局形貌拓扑重构实验, 以综合 验证基于足端位置的地形感知与表征方法。实验样 机 HEXA 采用典型的三关节六足圆周机体布置构 型, 机体内置可动态监测机体位姿的陀螺仪, 足端 加装用于识别支撑状态的压力传感器, 通过协同伺 服控制各关节实时转角能够以自由步态稳定漫游于 未知复杂地形, 主要技术参数如表 1 所示。实验地 形选用相对平缓的丘陵式地貌, 通过优化设计已充 分综合起伏、障碍等各类典型地形特征。三维光学 测量仪主要用于实验地形标定, 采用栅格化测量方 法可便捷获取实验地形标定形貌，以便与基于足端 位置构造的局部地形和全局形貌对比分析, 量化评 估方法地形表征重构精度性能。

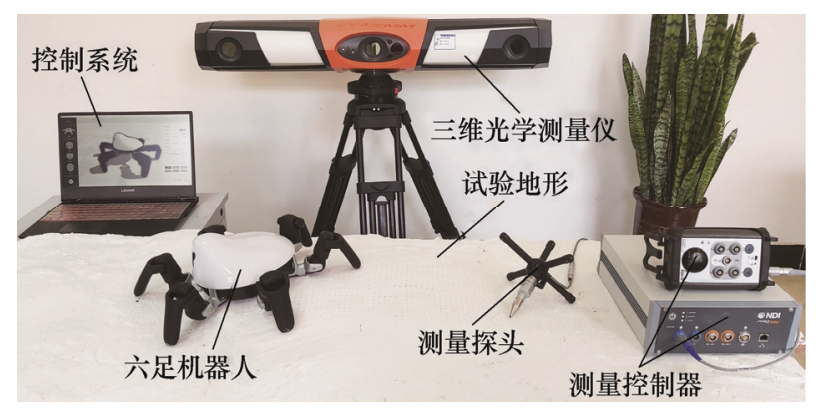

图 6 机器人实验平台
表 1 机器人主要技术参数

\begin{tabular}{cc||cc}
\hline 性能指标 & 技术参数 & 性能指标 & 技术参数 \\
\hline 机体半径 $/ \mathrm{mm}$ & 73 & 六足分布夹角 $/\left(^{\circ}\right)$ & 60 \\
基节长度 $/ \mathrm{mm}$ & 59 & 躯基关节转角 $/\left(^{\circ}\right)$ & $-45 \sim 45$ \\
股节长度 $/ \mathrm{mm}$ & 47 & 基股关节转角 $/\left(^{\circ}\right)$ & $-80 \sim 80$ \\
胫节长度 $/ \mathrm{mm}$ & 88 & 股胫关节转角 $/\left(^{\circ}\right)$ & $-35 \sim 135$ \\
\hline
\end{tabular}

实验中控制机器人以占空比约为 $3 / 4$ 的自由 步态沿直线缓慢漫游于实验地形, 如图 7 所示, 则基于时域内足端序列周期化处理机制, 依据足 端压力传感器反馈信息, 可将整个实验生成足端 序列离散为若干周期。综合考虑实验地形典型特 征和足端序列空间分布, 选取前 85 个周期实验过 程数据用于后续局部地形量化表征和全局形貌拓 扑重构的分析处理，以充分验证不同特征地形下 地形感知与表征方法的综合性能。选定周期内机 器人各足关节转角及其足端支撑状态均可由控制 系统导出, 具体分别如图 8 和图 9 所示。综合分 析图 8 和图 9 可知, 机器人各足关节转角均以 12 个周期为间隔呈现近似周期循环趋势, 其中摆动 相普遍占据 3 个周期, 支撑相通常跨越 9 个周期, 高度契合自由步态预设占空比，同时循环期间各 足对应周期关节转角均存在微量波动, 支撑状态 亦存在微小相位差异, 间接表明机器人以自由步 态漫游于非平坦实验地形过程中基于足端压力反 馈通过各足关节转角的自适应协同调整可确保支 撑足端与多变地形充分接触。

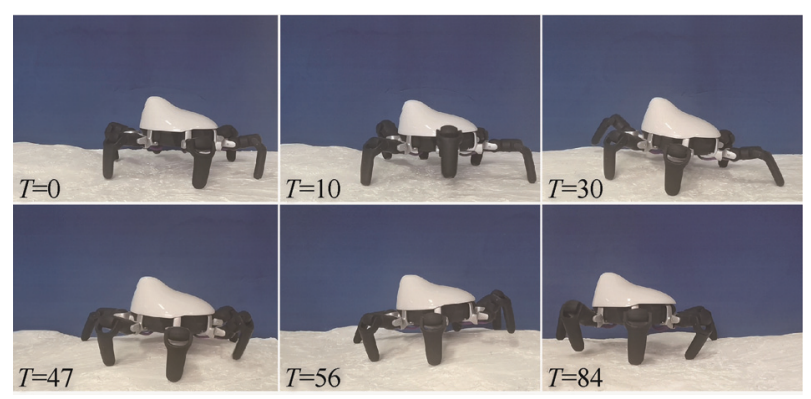

图 7 机器人漫游运动场景

实验观测发现, 受限于机体内置陀螺仪固有精 度特性及多变地形造成的振动冲击影响, 陀螺仪标 准欧拉角采样数据存在明显的随机误差。由于基于 标准欧拉角解算的机体位姿将作为局部地形量化表 征和全局形貌拓扑重构的重要基准, 因此精准校正 补偿标准欧拉角采样数据对提升地形感知与表征精 
度至关重要。考虑相邻周期足端位置状态中均存在 共用支撑足端, 以致其映射的微小局部地形间形貌 变化通常较为有限, 因而各周期标准欧拉角理论上 应随地形呈现较为平缓的连续变化趋势。为此, 借 鉴典型的邻域均值方法, 利用与之最为临近的 6 周 期采样数据校正补偿任一周期标准欧拉角, 选定周 期内标准欧拉角原始采样数据与校正补偿数据如图 10 所示。分析图 10 可知, 机体俯仰角 $\alpha_{n}$ 和横
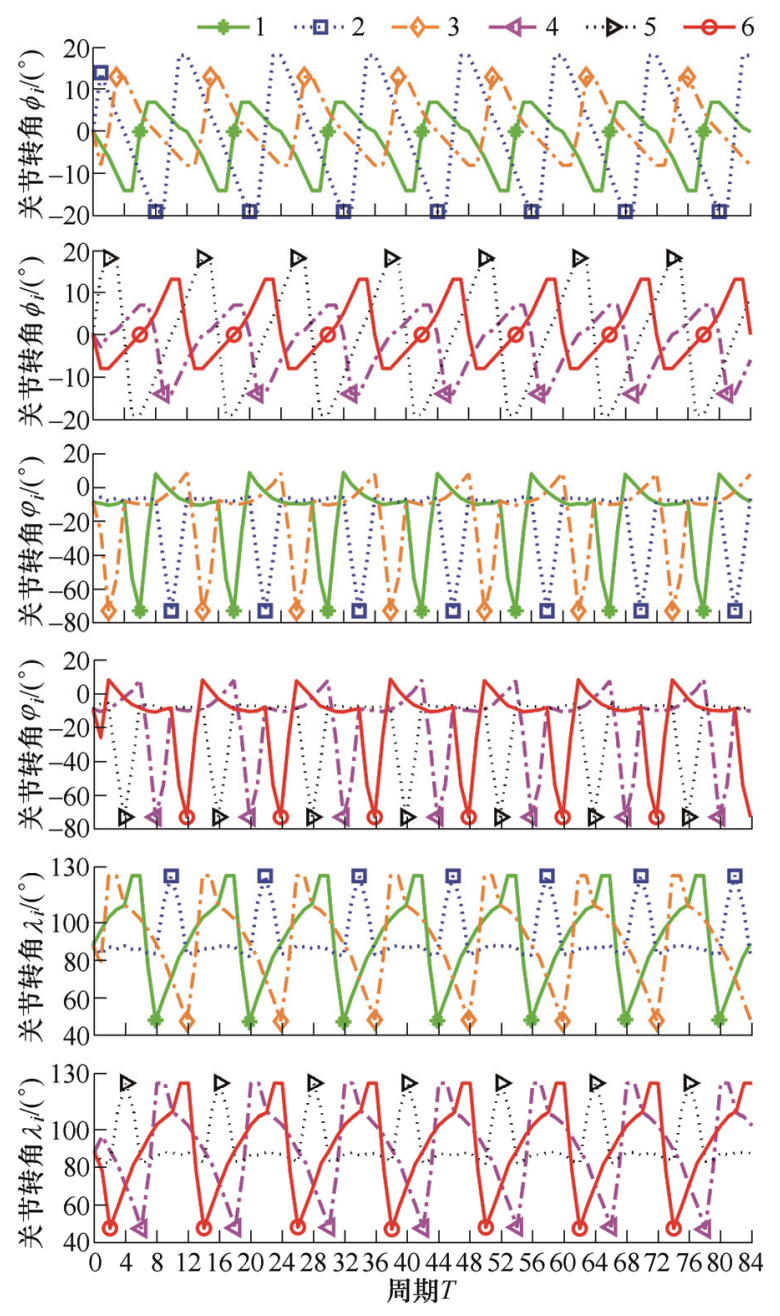

图 8 机器人关节实时转角

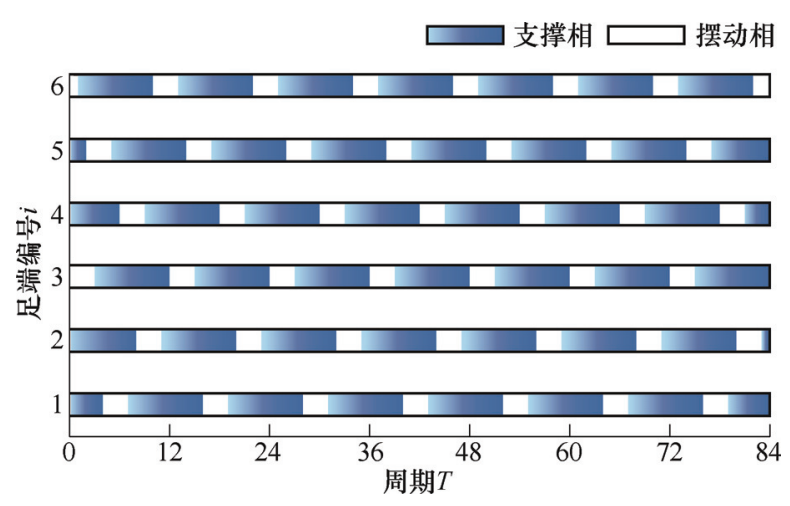

图 9 机器人足端支撑状态
滚角 $\beta_{n}$ 均存在明显的波动现象及随机误差, 且 $\alpha_{n}$ 尤为显著, 与实验地形在机体前进方向高程波动趋 势明显强于宽度方向事实高度相符，由于较大随机 误差多分布于高程波动剧烈处, 可知多变地形引发 的机体振动冲击系造成采样随机误差的主导因素, 同时机体偏航角 $\gamma_{n}$ 并无明显波动及误差，可间接表 明机器人以自由步态漫游于实验地形过程中能够精 准跟随预设直线运动轨迹。

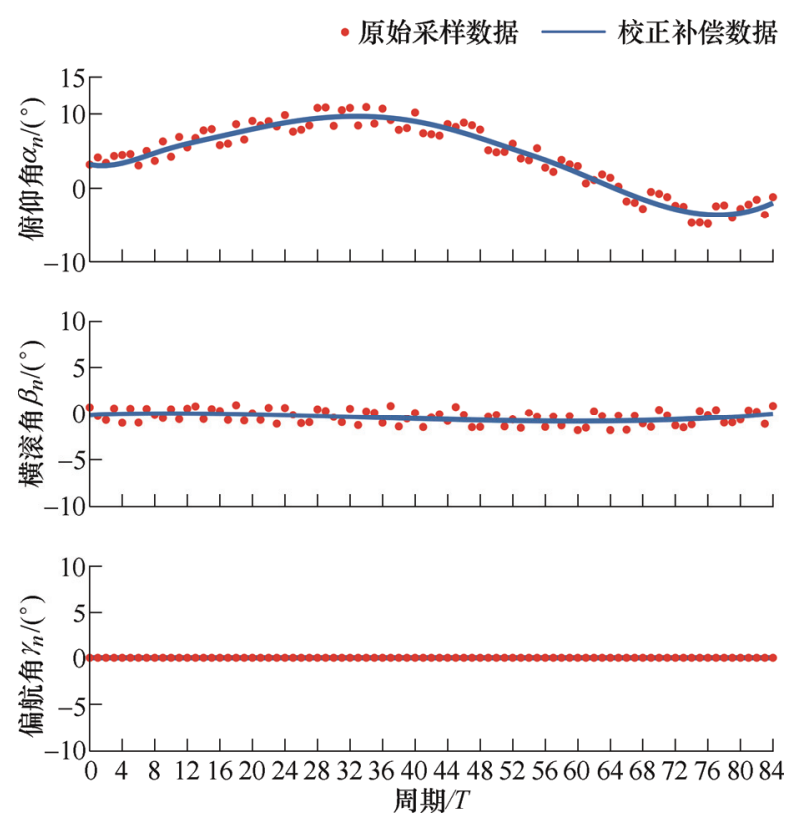

图 10 机体陀螺仪标准欧拉角数据

如前所述，局部地形量化表征与全局形貌拓 扑重构的关键在于如何基于无序足端位置状态在 时变机体坐标系下构造出可量化表征周期映射局 部地形的微小特征平面并将其以连续精准的机体 位姿作为参照基准统一转换至同一参考坐标系进 行合理拓扑拼接，因而其间需精准获悉各周期足 端位置状态及机体位姿信息。基于足端位置状态 的矢量化描述规则，各周期足端支撑状态均可依 据足端压力传感器反馈信息直接确定, 支撑足端 坐标亦可参照时变机体坐标系下足端位置解算模 型利用各足关节实时转角程式化求取。而各周期 机体位姿则需要结合共用支撑足端坐标和机体姿 态传感数据间接解算, 通过实验过程数据的预先 处理, 可获取各周期共用支撑足端坐标及机体标 准欧拉角参数如表 2 所示，其中共用足端依据相 邻周期足端位置矢量的参数对比结果随机选取, 足端坐标基于前置周期机体坐标系, 标准欧拉角 为校正补偿后参数。 
表 2 各周期共用支撑足端与机体标准欧拉角参数

\begin{tabular}{cccc}
\hline 周期 & 共用足端 & 足端坐标 & 标准欧拉角 \\
\hline 0 & 1 & $(96.11,170.84,-79.03)$ & $(3.18,-0.21,0)$ \\
1 & 4 & $(-95.69,-158.45,-79.16)$ & $(3.04,-0.13,0)$ \\
2 & 4 & $(-95.35,-169.53,-79.58)$ & $(3.04,-0.11,0)$ \\
3 & 4 & $(-97.26,-177.32,-79.99)$ & $(3.15,-0.09,0)$ \\
4 & 4 & $(-98.04,-188.72,-79.95)$ & $(3.37,-0.08,0)$ \\
5 & 4 & $(-97.25,-198.51,-79.07)$ & $(3.65,-0.07,0)$ \\
6 & 3 & $(-93.63,-148.73,-79.71)$ & $(3.99,-0.06,0)$ \\
$\vdots$ & $\vdots$ & $\vdots$ & $\vdots$ \\
29 & 4 & $(-97.25,-198.51,-79.83)$ & $(9.51,-0.32,0)$ \\
30 & 3 & $(93.63,-148.73,-79.21)$ & $(9.59,-0.34,0)$ \\
31 & 3 & $(94.05,-159.49,-79.61)$ & $(9.64,-0.37,0)$ \\
$\vdots$ & $\vdots$ & $\vdots$ & $\vdots$ \\
79 & 3 & $(94.05,-159.49,-79.57)$ & $(-3.52,-0.40,0)$ \\
80 & 3 & $(95.35,-169.53,-80.03)$ & $(-3.37,-0.34,0)$ \\
81 & 3 & $(95.29,-178.39,-79.91)$ & $(-3.15,-0.29,0)$ \\
82 & 3 & $(95.87,-189.80,-79.49)$ & $(-2.85,-0.22,0)$ \\
83 & 3 & $(94.91,-199.57,-79.68)$ & $(-2.47,-0.15,0)$ \\
84 & - & - & $(-2.01,-0.08,0)$ \\
\hline
\end{tabular}

鉴于实验地形已充分综合起伏、障碍等各类典 型地形特征, 利用机器人行经相应特征地形的对应 周期实验数据, 依次开展了不同特征局部地形的量 化表征实验, 以综合评估基于足端位置的局部地形 量化表征方法。局部平坦地形量化表征实验选用第 1 周期相关数据, 其结果如图 11 所示。图 $11 \mathrm{a}$ 为基 于足端位置矢量在初始参考坐标系下构建的局部地 形特征平面, 图 $11 \mathrm{~b}$ 为利用三维光学测量仪标定获 取的实验地形相应区域标定形貌, 图 11c 为局部地 形特征平面相对于精准标定形貌的整体高程误差分 布, 图 11d 为基于整体高程误差分布切片统计获取 的各轴向高程误差分布情况, 其中误差范围表象上 体现为整体高程误差分布的轴向投影, 其边界分别 为相应轴向误差分布切片的最大值和最小值, 平均 误差则为对应轴向误差分布切片的平均值。由图 11 可知, 基于足端位置矢量构建的局部平坦地形特征 平面法矢量 $\boldsymbol{n}$ 为 $(-0.0025,0.0046,-1)$, 平面方程参 数 $D$ 为 78.59 , 三个关键地形特征点分别为 $\widehat{G}_{1}^{1}(-62.80,20.22,-78.34) 、 \widehat{G}_{2}^{1}(-32.49,-22.49$, -78.62)、 $\widehat{G}_{3}^{1}(61.61,40.43,-78.56)$, 在参照关键地形 特征点位置分布选定的水平投影为 $60 \mathrm{~mm} \times 70 \mathrm{~mm}$ 对比区域内, 与精准标定形貌相比, 整体高程误差 分布数值较小且相对均衡, $y$ 轴向(机体前进方向) 和 $x$ 轴向 (机体宽度方向) 误差范围最大值分别为 $3.39 \mathrm{~mm}$ 和 $2.91 \mathrm{~mm}$, 最大平均误差分别为 $2.66 \mathrm{~mm}$ 和 $2.63 \mathrm{~mm}$ 。同理, 选用第 29 和第 79 周期相关数
据分别开展了局部上坡地形和局部下坡地形的量化 表征实验, 其结果分别如图 12 和图 13 所示。图 12 中构建的局部上坡地形特征平面法矢量 $\boldsymbol{n}$ 为 $(0.0073$, $0.1029,-1)$, 平面方程参数 $D$ 为 74.65 , 关键地形特 征点分别为 $\widehat{G}_{1}^{29}(-28.93,256.10,-48.52) 、 \widehat{G}_{2}^{29}(1.80$, $298.49,-43.93) 、 \widehat{G}_{3}^{29}(65.75,318.29,-41.43)$, 整体高 程误差分布较为合理均衡, $y$ 轴向和 $x$ 轴向误差范 围最大值分别为 $5.73 \mathrm{~mm}$ 和 $4.39 \mathrm{~mm}$, 最大平均误 差分别为 $2.78 \mathrm{~mm}$ 和 $2.69 \mathrm{~mm}$ 。图 13 中构建的 局部下坡地形特征平面法矢量 $\boldsymbol{n}$ 为 $(0.0041,-0.1126,-1)$, 平面方程参数 $D$ 为 -15.03 , 关键 地形特征点分别为 $\widehat{G}_{1}^{79}(-54.10,817.11,-77.23)$ $\widehat{G}_{2}^{79}(39.11,753.34,-69.66) 、 \widehat{G}_{3}^{79}(69.89,795.37$, -74.27), 整体高程误差分布亦较为理想均衡, $y$ 轴 向和 $x$ 轴向误差范围最大值分别为 $4.08 \mathrm{~mm}$ 和 $3.85 \mathrm{~mm}$, 最大平均误差分别为 $2.97 \mathrm{~mm}$ 和 $2.87 \mathrm{~mm}$ 。 综合分析上述实验结果可知, 尽管实验选取的局部 地形形貌存在幅度较为明显的随机波动, 但基于地 形特征点迭代篩选规则, 通过采用遍历组合和隐含 加权方式充分综合各周期足端位置状态信息，构造 出的不同特征局部地形特征平面整体高程误差分布 均较为理想均衡, 特征平面 $y$ 轴向误差普遍高于 $x$ 轴向，且局部上坡和局部下坡地形各轴向误差均整 体高于局部平坦地形，间接表明地形形貌波动幅度 与地形表征精度间存在较为明显的负相关关系, 此 外考虑大量稠密的周期位置状态实际映射的局部地 形区域远小于实验选定区域，局部地形量化表征精 度实质上将大幅高于上述实验数值。实验结果表明, 基于足端位置的局部地形量化表征方法通过制定合 理可行的地形特征点迭代篎选规则充分综合各周期 无序足端位置状态信息, 利用程式化遴选出的关键地 形特征点构建的周期映射局部地形特征平面能够在 较高精度要求下合理量化表征不同特征局部地形。

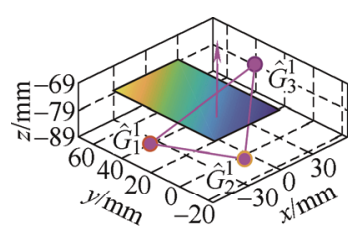

(a)

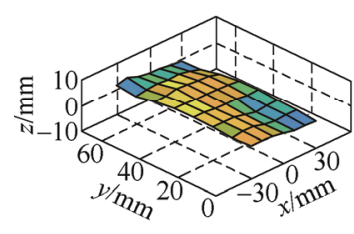

(c)

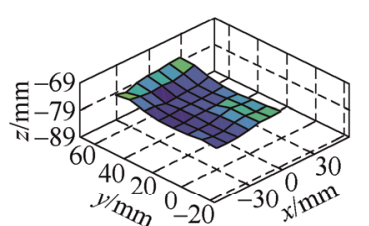

(b)

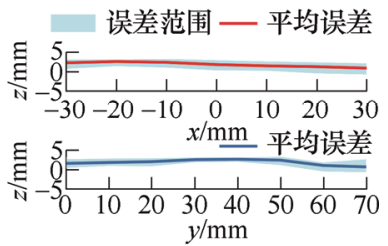

(d)
图 11 局部平坦地形量化表征实验结果 


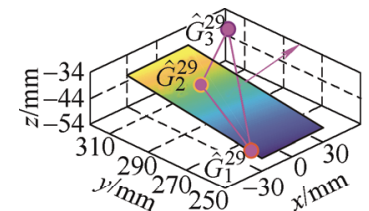

(a)

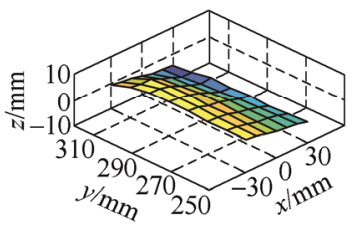

(c)

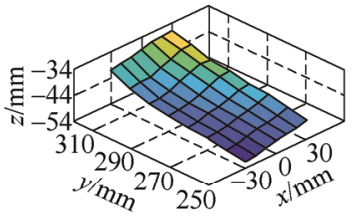

(b)

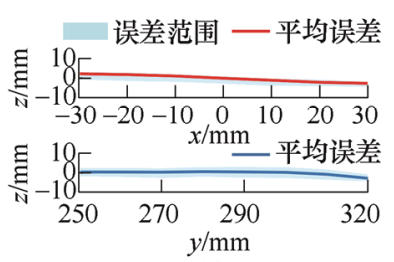

(d)
图 12 局部上坡地形量化表征实验结果

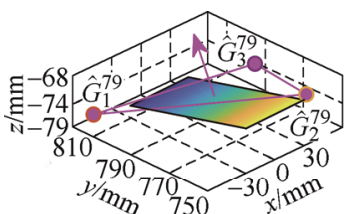

(a)

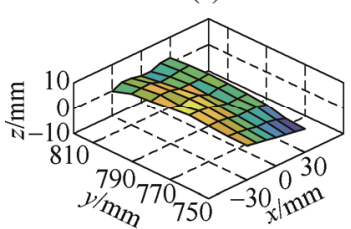

(c)

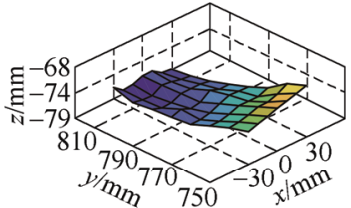

(b)

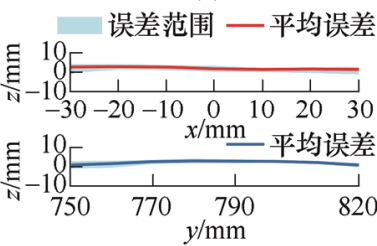

(d)
图 13 局部下坡地形量化表征实验结果

为综合验证基于机体位姿的全局形貌拓扑重构 方法, 参照基于共用支撑足端坐标和机体姿态传感 数据构建的各周期机体位姿间变换关系, 将时变机 体坐标系下构造的前 85 个周期地形特征平面统一 转换至初始参考坐标系, 并依据特征平面拓扑拼接 规则对其按照时序依次裁剪拼接, 设计开展了全局 形貌拓扑重构实验, 其结果如图 14 所示。图 14a 为基于连续精准机体位姿参照拓扑重构出的漫游地 形全局形貌, 图 $14 \mathrm{~b}$ 为利用三维光学测量仪标定获 取的实验地形相应区域全局形貌，图 14c 为重构全 局形貌相对于精准标定全局形貌的整体高程误差分 布, 图 14d 为基于整体高程误差分布切片统计获取 的各轴向高程误差分布情况。由图 14 可知, 重构全 局形貌整体高程误差分布较为理想均衡, $y$ 轴向和 $x$ 轴向误差范围最大值分别为 $7.11 \mathrm{~mm}$ 和 $6.98 \mathrm{~mm}$, 最大平均误差分别为 $3.28 \mathrm{~mm}$ 和 $2.52 \mathrm{~mm}$, 明显优 于传统全局形貌拓扑重构方法(基于激光雷达、双目 相机等外部传感器件)通常具备的 $10 \mathrm{~mm}$ 左右表征

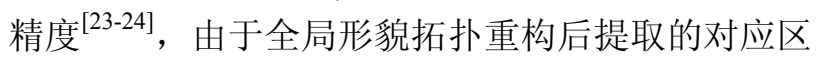
域局部地形表征精度略优于上述局部地形量化表征 实验结果, 可知依据拓扑拼接规则裁剪获取的微小 特征平面能够更为精准的表征各周期映射局部地
形, 且伴随漫游进程的持续开展, 利用丰富的新增 足端位置状态序列将可持续提升全局形貌拓扑重构 精度, 同时 $y$ 轴向误差范围和平均误差均相对高于 $x$ 轴向, 且较大误差多分布于形貌波动剧烈处, 再 次间接印证地形形貌波动幅度与地形表征精度间存 在较为明显的负相关关系。实验结果表明基于机体 位姿的全局形貌拓扑重构方法通过构建各周期机体 位姿间变换关系, 以连续精准机体位姿作为参照基 准, 将时变机体坐标系下构造的各周期地形特征平 面统一转换至同一参考坐标系, 并依据特征平面拓 扑拼接规则进行合理裁剪拼接, 能够在较高精度要 求下重构表征机器人漫游地形全局形貌。

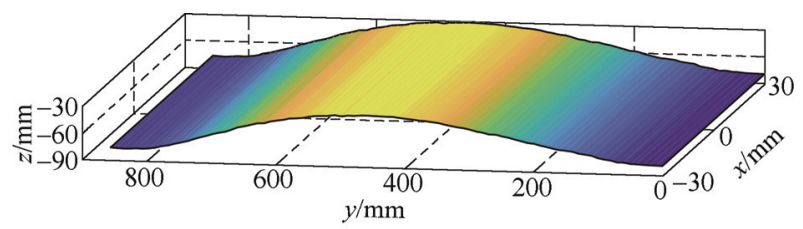

(a)

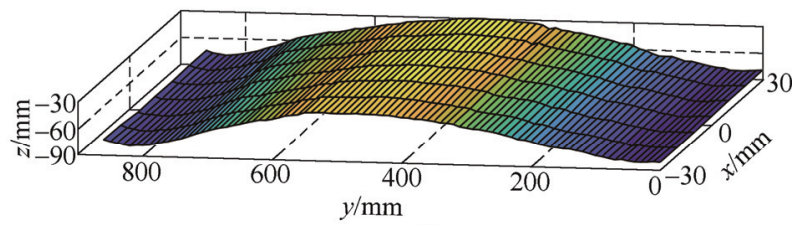

(b)

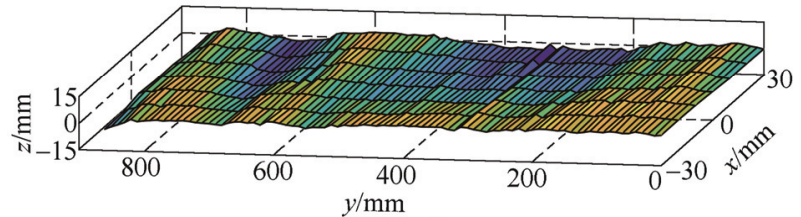

(c)
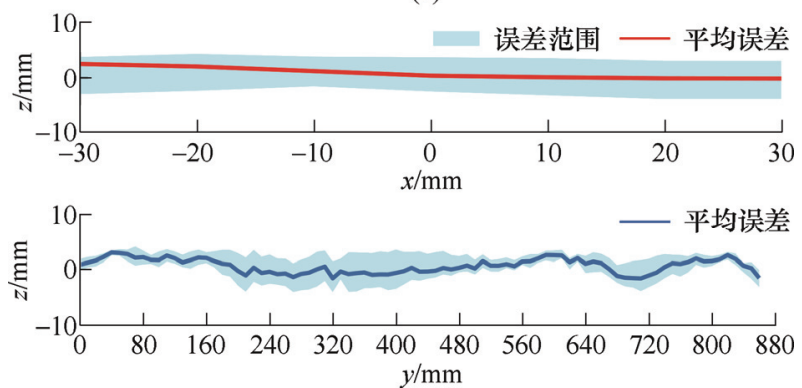

(d)

图 14 全局形貌拓扑重构实验结果

\section{5 结论}

（1）基于机器人构型分析与结构约束，采用矢 量法构建时变机体坐标系下足端位置解算模型，利 用丰富无序的支撑足端坐标量化表征机器人漫游地 形, 能够在一定程度上初步解决传统基于外部传感 的地形感知与表征方法普遍存在的感知范围局限、 感知精度不足、表征效果欠佳等问题。 
（2）基于无序足端位置状态序列的周期化处理 与矢量化描述, 通过制定合理可行的地形特征点迭 代篮选规则, 采用遍历组合和隐含加权方式充分综 合各周期足端位置信息, 利用程式化遴选出的关键 地形特征点构建的微小地形特征平面能够在较高精 度要求下合理量化表征周期映射局部地形。

（3）基于时变机体位姿间耦合约束与变换机制 深度剖析, 利用共用支撑足端坐标和机体姿态传感 数据构建相邻周期机体位姿间递推关系, 以连续精 准的机体位姿作为参照基准, 将时变机体坐标系下 构造的各周期地形特征平面统一转换至同一参考坐 标系, 并制定合理可行的特征平面拓扑拼接规则按 照时序依次裁剪拼接, 能够在较高精度要求下重构 表征机器人漫游地形全局形貌。

（4）实验结果表明, 相比传统基于外部传感的 地形感知与表征方法, 基于足端位置的六足机器人 地形感知与表征方法能够在无需增设外部观测传感 器件条件下较为精准合理的量化表征不同特征局部 地形, 并实现漫游地形全局形貌的精准拓扑重构。

\section{参 考 文 献}

[1] ZHONG Bin, ZHANG Shiwu, XU Min, et al. On a CPG-based hexapod robot: AmphiHex-II with variable stiffness legs[J]. IEEE/ASME Transactions on Mechatronics, 2018, 23(2): 542-551.

[2] NGUYEN C $\mathrm{T}$, PHUNG $\mathrm{H}$, HOANG $\mathrm{P} \mathrm{T}$, et al. Development of an insect-inspired hexapod robot actuated by soft actuators[J]. Journal of Mechanisms and Robotics, 2018, 10(6): 1-8.

[3] BJELONIC M, KOTTEGE N, HOMBERGER T, et al. Weaver: hexapod robot for autonomous navigation on unstructured terrain[J]. Journal of Field Robotics, 2018, 35(7): 1063-1079.

[4] LEE Y, LEE H, HWANG S, et al. Terrain edge detection for biped walking robots using active sensing with vCoP-position hybrid control[J]. Robotics and Autonomous Systems, 2017, 96(1): 41-57.

[5] HUANG Hai, TANG Qirong, LI Jiyong, et al. A review on underwater autonomous environmental perception and target grasp, the challenge of robotic organism capture[J]. Ocean Engineering, 2020, 195(1): 1-11.

[6] MARTINEZ-HERNANDEZ U, RUBIO-SOLIS A , PRESCOTT T J. Learning from sensory predictions for autonomous and adaptive exploration of object shape with a tactile robot[J]. Neurocomputing, 2020, 382(1) :
127-139.

[7] NEFTI-MEZIANI S, MANZOOR U, DAVIS S, et al. 3D perception from binocular vision for a low cost humanoid robot NAO[J]. Robotics and Autonomous Systems, 2015, 68(1): 129-139.

[8] ZHAO Xu, LIU Chang, DOU Lihua, et al. 3D visual sensing technique based on focal stack for snake robotic applications[J]. Results in Physics, 2019, 12(1) : 1520-1528.

[9] HADDELER G, AYBAKAN A, AKAY M C, et al. Evaluation of 3D lidar sensor setup for heterogeneous robot team[J]. Journal of Intelligent and Robotic Systems: Theory and Applications, 2020, 100(2): 689-709.

[10] EINHORN E, SCHRTER C , GROSS H M. Attention-driven monocular scene reconstruction for obstacle detection, robot navigation and map building $[\mathrm{J}]$. Robotics and Autonomous Systems, 2011, 59(5): 296-309.

[11] LI Ge, ZHANG Xuehe, LI Changle, et al. Design and application of parallel stereo matching algorithm based on CUDA[J]. Microprocessors and Microsystems, 2016, 47(1): $142-150$

[12] 彭建建, 白瑞林. 基于水平树结构的可变权重代价聚合 立体匹配算法[J]. 光学学报, 2018, 38(1): 214-221.

PENG Jianjian, BAI Ruilin. Variable weight cost aggregation algorithm for stereo matching based on horizontal tree structure[J]. Acta Optica Sinica, 2018, 38(1): 214-221.

[13] LOMBARD C D, VAN D. Stochastic triangular mesh mapping: A terrain mapping technique for autonomous mobile robots[J]. Robotics and Autonomous Systems, 2020, 127(1): 1-27.

[14] 孙宇, 项志宇, 刘济林. 未知室外环境下移动机器人的 三维场景重建 [J]. 浙江大学学报, 2007(12): 1949-1954. SUN Yu，XIANG Zhiyu, LIU Jilin. 3D map building for mobile robot under unknown outdoor environments $[\mathrm{J}]$. Journal of Zhejiang University, 2007(12): 1949-1954.

[15] STUECKLER J, SCHWARZ M, SCHADLER M, et al. NimbRo explorer : Semiautonomous exploration and mobile manipulation in rough terrain[J]. Journal of Field Robotics, 2016, 33(4): 411-430.

[16] 张慧, 荣学文, 李贻斌, 等. 四足机器人地形识别与路 径规划算法 $[J]$. 机器人, 2015, 37(5): 546-556.

ZHANG Hui, RONG Xuewen, LI Yibin, et al. Terrain recognition and path planning for quadruped robot $[\mathrm{J}]$. Robot, 2015, 37(5): 546-556.

[17] BELTER D, LABECKI P, SKRZYPCZYNSKI P. 
Estimating terrain elevation maps from sparse and uncertain multi-sensor data[C]// 2012 IEEE International Conference on Robotics and Biomimetics, December 11-14, 2012, Guangzhou, China. Washington D.C.: IEEE Computer Society, 2012: 715-722.

[18] FANKHAUSER P, BLOESCH M, HUTTER M. Probabilistic terrain mapping for mobile robots with uncertain localization[J]. IEEE Robotics and Automation Letters, 2018, 3(4): 3019-3026.

[19] 陈栋, 李世其, 王峻峰, 等. 并联机构的运动学多目标 轨迹规划方法 $[J]$. 机械工程学报, 2019，55(15): 163-173.

CHEN Dong, LI Shiqi, WANG Junfeng, et al. Method of multi-objective trajectory planning of parallel mechanism based on the kinematics[J]. Journal of Mechanical Engineering, 2019, 55(15): 163-173.

[20] 李研彪, 郑航, 孙鹏, 等. 考虑关节摩擦的 5-PSS/UPU 并联机构动力学建模及耦合特性分析 $[\mathrm{J}]$. 机械工程学 报，2019，55(3): 43-52.

LI Yanbiao, ZHENG Hang, SUN Peng, et al. Dynamic modeling with joint friction and research on the inertia coupling property of a 5-PSS/UPU parallel manipulator[J]. Journal of Mechanical Engineering, 2019， 55(3): 43-52.

[21] AGHELI M, NESTINGER S S. Force-based stability margin for multi-legged robots[J]. Robotics and Autonomous Systems, 2016，83(1): 138-149.
[22] 李满宏, 张明路, 张建华, 等. 基于增强学习的六足机 器人自由步态规划[J]. 机械工程学报, 2019, 55(5): 36-44.

LI Manhong, ZHANG Minglu, ZHANG Jianhua, et al. Free gait planning for a hexapod robot based on reinforcement learning $[\mathrm{J}]$. Journal of Mechanical Engineering, 2019, 55(5): 36-44.

[23] DROESCHEL D , SCHWARZ M , BEHNKE S. Continuous mapping and localization for autonomous navigation in rough terrain using a 3D laser scanner[J]. Robotics and Autonomous Systems, 2017, 88(1): 104-115.

[24] BELTER D, LABECKI P, FANKHAUSER P, et al. RGB-D terrain perception and dense mapping for legged robots[J]. International Journal of Applied Mathematics and Computer Science, 2016, 26(1): 81-97.

作者简介: 张明路, 男, 1964 年出生, 博士, 教授, 博士研究生导师。 主要研究方向为智能机器人技术。

E-mail: zhangml@hebut.edu.cn

王哲, 男, 1996 年出生, 硕士研究生。主要研究方向为六足机器人地形 感知与表征方法。

E-mail: wangzhe_stu@126.com

李满宏(通信作者), 男, 1987 年出生, 博士, 副教授, 博士研究生导师。 主要研究方向为六足机器人步态规划与运动控制方法。

E-mail:1mh9181219@163.com

张建华, 男, 1979 年出生, 博士, 教授, 博士研究生导师。主要研究方 向为柔性关节机器人柔顺控制方法。

E-mail: jhzhang@hebut.edu.cn

陈俊杰, 男, 1995 年出生, 硕士研究生。主要研究方向为六足机器人步 态规划。

E-mail: cjj9727@163.com 